Article

\title{
Assessment of Sustainability Indicators for Biodiesel Production
}

\author{
Edith Martinez-Guerra ${ }^{1,2}$ and Veera Gnaneswar Gude ${ }^{1, *}$ \\ 1 Department of Civil and Environmental Engineering, Mississippi State University, Starkville, MS 39759, \\ USA; EDITH.L.MARTINEZ-GUERRA@usace.army.mil \\ 2 Environmental Laboratory, Engineer Research and Development Center, Vicksburg, MS 39180, USA \\ * Correspondence: gude@cee.msstate.edu or gudevg@gmail.com; Tel.: +1-662-325-0345
}

Received: 31 July 2017; Accepted: 22 August 2017; Published: 25 August 2017

Featured Application: Sustainability Indicator Concepts used for biodiesel production in this study can be applied to various biofuels and other chemical reaction designs, synthesis and process development.

\begin{abstract}
Biodiesel production may provide a sustainable route to reduce environmental pollution caused by fossil fuel consumption. In order to minimize environmental impacts of biodiesel production, the chemical process should be optimized to minimize waste generation and energy consumption. Therefore, it is important to design biodiesel chemical reactions and processes using green chemistry and green engineering principles to develop sustainable chemical processes. This study provides the results of the synergistic effect of microwave and ultrasound irradiations to produce biodiesel using ethanol or methanol as the alcohol donor. The biodiesel yields are presented along with their respective green metrics, such as atom economy, environmental factor (E-factor), atom economy (utilization) or atomic efficiency, mass intensity, reaction mass efficiency, atom utilization, and stoichiometric factor. These green metrics are crucial to determine the sustainability and environmental impact of biodiesel production. Evaluation of these green metrics indicates that methanol is a better alternative for biodiesel production provided it is derived from renewable sources. Sustainability indicator concepts used for biodiesel production in this study can be applied to various biofuels and other chemical reaction designs, synthesis and process development.
\end{abstract}

Keywords: biodiesel; green chemistry; microwave; ultrasound; green metrics; atom economy; environmental factor (E-factor); mass intensity; reaction mass efficiency; stoichiometric factor

\section{Introduction}

In recent years, there has been a concerted effort across the scientific community to produce biofuels in an environmentally friendly and sustainable manner [1-3]. Green chemistry and Green Engineering principles have been adopted to achieve this goal. These two sets of principles are primarily oriented towards reaction design and optimization with respect to materials and energy usage, waste reduction and economic feasibility. These principles also focus on minimal use of toxic and hazardous chemicals and maximum safety practices. These principles are commonly applied to evaluate the performances of individual chemical reactions, chemical processes and chemical synthesis design and plans in various chemistry, chemical and process engineering disciplines. The fundamental concept for both green chemistry and green engineering is that ongoing efforts to improve reactions are directly connected to the optimization of key parameters that govern their performances. It is obvious that some of these parameters will need to be maximized and others minimized [3]. This research article focuses on the use of green chemistry metrics for evaluating a biodiesel production process. 
In order to be able to control chemical processes, Anastas and Warner introduced the concept of green chemistry along with the twelve green chemistry principles: (1) Prevention; (2) atom economy; (3) less hazardous chemical syntheses; (4) design safer chemicals; (5) safer solvents and auxiliaries; (6) design for energy efficiency; (7) use renewable feedstocks; (8) reduce derivatives; (9) catalysis; (10) design for degradation; (11) real-time analysis for pollution prevention; (12) inherently safer chemistry for accident prevention [4]. Green chemistry, in general, incorporates a new approach to the synthesis, processing and application of chemical substances in such a manner as to reduce threats to health and the environment. This new approach is also known as environmentally benign chemistry; clean chemistry; atom economy; and benign-by-design chemistry [5]. The concept of green chemistry refers to the design of chemical products and processes that minimize or eliminate the generation of hazardous substances and that seek to prevent or reduce the risk to human health and the environment [1]. Thus, green chemistry evaluates the greenness of a chemical process and allows to compare the greenness of existing solutions with newly developed ones [6]. Correspondingly, with green chemistry, the green chemistry metrics were introduced as metrics that measure aspects of chemical processes based on the twelve aforementioned green chemistry principles. Green metrics have proved to be a simple and versatile method for an immediate evaluation of the sustainability of a chemical process of any kind.

Different components must be taken into account when seeking the sustainability of competing chemical reactions; these should include the toxicity of a product, reaction conditions, reagents, and reaction types. The most common metrics used to determine the sustainability of a chemical process are atom economy [7], and the E-factor [7-11]. These are simple calculations that mainly focus on waste, except for effective mass yields that focus on the toxicity of reagents. In addition to the aforementioned metrics, several other green chemistry metrics will be discussed within this study including atom efficiency [7], mass intensity [8,12], mass productivity [8], reaction mass efficiency, atom utilization, and stoichiometric factor [3]. This study attempts to use green metrics calculations to determine the greenness of biodiesel production using waste cooking oil. Each individual metric is explained in detail within the materials and methods sections. Green chemistry metrics to calculate the sustainability of a product or a chemical process have been used mostly in the pharmaceutical industry $[13,14]$. Additionally, green metrics have been used in several other processes such as the production of certain electronic devices [12]. No previous or current experimental data has been reported for the application of green chemistry metrics in biodiesel production. Therefore, the main focus of this study is to report the findings of the application of green chemistry metrics to the synergistic effect of microwave and ultrasound irradiations in the conversion of waste cooking oil into biodiesel. These metrics can be used in any chemical process implemented for biofuel production.

\section{Materials and Methods}

\subsection{Materials}

The waste cooking oil (WCO) was obtained from the Mississippi State University cafeteria. The acid value of $\mathrm{WCO}$ was found to be $3.5 \mathrm{mg} \mathrm{KOH} / \mathrm{g}$, corresponding to a free fatty acid (FFA) level of $1.7 \%$. From the Gas Chromatograph-flame ionization detector (GC-FID), the molecular weight for the waste cooking oil was $840 \mathrm{~g} / \mathrm{mol}$. The alcohols (ethanol and methanol) and the catalyst are of analytical grade and were purchased from Fisher Scientific (Hampton, NH, USA). A microwave/ultrasound reactor unit and three-neck custom-fabricated reaction vessels made of borosilicate glass were manufactured by Columbia International Technologies ${ }^{\circledR}$ (Calgary, AB, Canada).

\subsection{Methods}

Various conditions involved in transesterification reaction were evaluated through a mechanistic study. These include reaction time, catalyst amount, methanol to oil molar ratio and oil volume ratios. The tested conditions include reaction time for 1-4 min with increments of $1 \mathrm{~min}$; a methanol to oil 
molar ratio of $4.5: 1$ to $12: 1$; catalyst concentrations ranging from $0.50-1.25 \%$; and an equal power output for microwave and ultrasound $(100 / 100 \mathrm{~W} / \mathrm{W})$. The oil volume was fixed to $20 \mathrm{~mL}$ and was added to a homogenous mixture of different weight percentages of $\mathrm{NaOH}$ and different alcohol volumes. For example, a $6: 1$ alcohol to oil ratio was equivalent to $6.15 \mathrm{~mL}$ of methanol or $8.35 \mathrm{~mL}$ of ethanol for $20 \mathrm{~mL}$ of oil; all calculations are based on molecular weight and the specific gravity of each alcohol and the oil. However, when studying different oil volumes, the alcohol to oil ratio was fixed to 6:1, catalyst amount was $0.75 \%(w t / w t)$, and the combined power output was $400 \mathrm{~W}$ (200 $\mathrm{W}$ for min reaction time). Two layers of product were derived after each test. The two layers-biodiesel and the glycerol-were separated and were both measured to determine green metrics. The benefits of combining microwave and ultrasound effects in biofuel production have been discussed elsewhere [15]. In addition, details of process parametric evaluation studies focusing on microwave and ultrasound effects, alcohol and catalyst effect on transesterification of waste cooking oils can be found in our previous studies [16-18]. Experiments conducted in this study were intended to evaluate the green metrics of the microwave and ultrasound-enhanced biodiesel production.

\subsection{Evaluation of Sustainability Indicators}

\subsubsection{Environmental Factor (E-factor)}

The E-factor is a simple and fast metric, and is the most frequently used metric to measure the potential environmental acceptability of a chemical process. First introduced by Roger Sheldon [11], the E-factor is expressed as the ratio (equation) of the actual total amount of waste to the desired product (Equation (1)), excluding water because it will lead to high E-factors which are not useful for comparing processes [7-11]. A higher E-factor implies that an excess amount of waste is generated, and therefore, the risk of negative environmental impact is higher. Hence, an ideal $E$-factor is zero meaning that no waste was generated during the process.

$$
E-\text { factor }=\frac{\text { Total waste }(\mathrm{kg})}{\text { Product }(\mathrm{kg})}
$$

\subsubsection{Atom Economy $(A E)$ and Atom Efficiency}

Atom economy has been considered as a measure of environmental sustainability in minimizing the amount of theoretical waste [19] as shown in Equation (2). This concept was introduced by Barry M. Trost at Stanford University in 1991, and it depends on the molecular weights of the reactants and the final product. It was initially proposed to avoid relying only on the yield of a product to measure its greenness. Unfortunately, the usefulness of $A E$ is limited since it only considers the stoichiometry of the reaction and does not take into account the yield of the desired product [20]. The ideal atom economy for a chemical transformation is taken as the process where all reactant atoms are found in the desired product [19]. Since the catalyst concentration for biodiesel production was insignificant and consumed during the reaction, it is excluded from the $A E$ calculations. Similarly, atom efficiency (Equation (3)) is the product of atom economy and the obtained percentages yield from the reaction. Atom economy provides the actual efficiency and utilization of atoms to produce useful products [1].

$$
A E=\frac{\text { MW of product } C}{M W \text { of } A+M W \text { of } B} \times 100
$$

On the other hand, atom efficiency provides a more concise solution because it also takes into account the production yield percentage.

$$
\text { Atom efficiency }=A E \times \% \text { yield }
$$


2.3.3. Mass Intensity $(M I)$ and Mass Productivity $(M P)$

Also known as process mass intensity (PMI), mass intensity (MI) was proposed by Constable et al. [8]. MI, as shown in Equation (4), is defined as the total mass used in a process divided by the mass of the product. Mass used in the process includes reagents, solvents, and catalyst. $M I$ can also be calculated as the E-factor plus one; therefore, the ideal $M I$ is $1(\mathrm{~kg} / \mathrm{kg})$ with zero for the $E$-factor. Equation (5) is used to calculate mass productivity, which is just the reciprocal of the $M I$; thus, MP depends merely on MI. Also, $M I$ is the preferred metric to drive greater efficiencies in pharmaceutical syntheses [14].

$$
\begin{gathered}
M I=\frac{\text { Total mass used in a process }(\mathrm{kg})}{\text { Mass of final product }(\mathrm{kg})} \\
M P=\frac{1}{M I} \times 100
\end{gathered}
$$

\subsubsection{Reaction Mass Efficiency or Material Efficiency $(R M E)$}

RME has been emphasized as a realistic metric for describing the greenness of a process [21]. It offers a better and easy way of identification of the best or worst reactions that have influence on the whole industrial process or synthesis [6]. RME takes into account yield, stoichiometry, and atom economy, and it is related to the E-factor by an inverse expression. According to Jimenez-Gonzalez et al. [14], $P M$ and $R M E$ are an indispensable intermediate step to estimate life cycle impacts and footprints. There are several $R M E$ equations, which depend on the conditions of the reaction process; for this study, Equations (7) and (8) have been used to calculate the RME of WVO transesterification. Ideally, $R M E$ for a reaction involving reactants of $\mathrm{A}$ and $\mathrm{B}$ to yield products $\mathrm{C}$ and D, as shown in Equation (6), can be presented as Equations (7) and (8).

$$
\begin{gathered}
A+B \rightarrow C+D \\
R M E=\frac{\text { Mass of product }(C+D)(\mathrm{kg})}{\text { Mass of } A(\mathrm{~kg})+\text { mass of } B(\mathrm{~kg})}
\end{gathered}
$$

or

$$
R M E=\frac{1}{1+E_{f}}
$$

\subsubsection{Atom Utilization}

$A U$ provides a fast and simple evaluation reaction in terms of produced waste; however, solvents are excluded from calculations [7].

$$
A U \%=\frac{\text { Mass of the final product }}{\text { Total mass of all substances produced }}
$$

\subsubsection{Solvent and Catalyst Environmental Impact Parameter $(f)$}

This parameter has a value of zero only if all materials used in the process are recycled, recovered, or eliminated; otherwise, $f>0[3,22,23]$

$$
f=\frac{\sum \text { mass of reactants and postreaction solvents and materials }+ \text { mass of catalyst used }}{\text { Mass of final product }}
$$

\subsubsection{Stoichiometric Factor}

The stoichiometric factor $(S F)$ is a green metric that accounts for chemical reactions run under nonstoichiometric conditions, with one or more reagents in excess [22]. According to Andraos [22], $S F$ 
equal to unity means that the reaction is operated under stoichiometric conditions; otherwise, $S F$ is greater than $1[6]$.

$$
S F=1+\frac{(A E) \sum \text { Mass of excess } \frac{\text { reagents }}{\text { chemicals }}}{\text { Expected product mass at } 100 \% \text { yield }}
$$

The appendix shows sample calculations for evaluating these metrics using the results obtained from our experimental studies.

\section{Results and Discussions}

This section discusses the results from the evaluation of green chemistry metrics for biodiesel production under the synergistic effect of microwave and ultrasound using ethanol and methanol as solvents. The most common method of biodiesel production is by transesterification (alcoholysis) of oils (triglycerides) with methanol in the presence of a catalyst [24,25] which is shown in Figure 1 [26]. The stoichiometric reaction requires 1 mole of triglyceride and 3 moles of an alcohol solvent. However, excess alcohol is used to drive the reversible reaction forward in order to increase the yields of the alkyl esters and to assist phase separation from the glycerol.

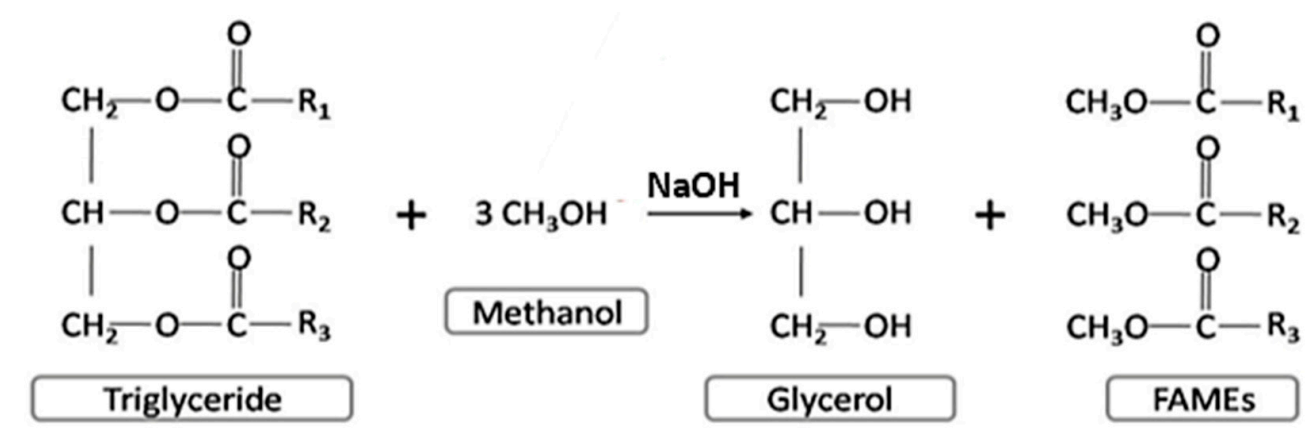

Figure 1. Reaction scheme for transesterification of triglycerides, modified from [26].

According to the twelve principles of green chemistry, a green solvent should meet numerous criteria such as low toxicity, non-flammability, non-mutagenicity, non-volatility and widespread availability among others. Moreover, these green solvents have to be cheap and easy to handle and recycle [27]. Here, we considered ethanol as a solvent due to its renewable characteristics. Producing biodiesel from low quality feedstock such as waste cooking oils is a challenging task due to the presence of high free fatty acid (FFAs) content. In addition, use of homogeneous catalysts can cause serious separation issues [28]. However, use of heterogeneous catalysts requires high temperatures and possibly high pressures with extended reaction times. Production of heterogeneous catalysts is also not a simple task. Cheap materials such as egg shells and other carbon-based catalysts can be used for this purpose [29] but this is not the focus of this research. For these reasons, we have considered the process intensification effects of microwave and ultrasound irradiations with the homogeneous catalyst-mediated transesterification reaction.

\subsection{Environmental Factor (E-factor)}

The E-factor was calculated assuming glycerol was a waste; however, a lower E-factor can be obtained if both biodiesel and glycerol are taken as products. Hence, all the calculations for each metric were done making the same assumption and taking biodiesel as the only main product. The E-factor was calculated for each condition when producing biodiesel using waste cooking oil and methanol or ethanol as the reactant. For each condition, it was noticed (Figure 2) that methanol has a lower E-factor, which means that less waste was produced when using methanol as a reactant. However, it is known that ethanol is an "environmental friendly" alcohol because it can be produced from renewable material. However, it was noticed that higher amounts of soap were present in the reaction, which was 
higher when compared to the use of methanol. This implies that the environmental factor is able to calculate or to predict which process (or material) will create more waste. Additionally, it can be observed that the E-factor is almost zero for a higher methanol to oil molar ratio, but it is higher when increasing the catalyst concentration. This can be attributed to a high content of free fatty acids present in the waste cooking oil, which increases the soap formation as the catalyst concentration increases. The E-factor for different oil volumes was influenced by the process intensification effect of microwave and ultrasound at a fixed power supply which is defined as power density. Under this condition, the E-factor for ethanol reactions was lower because ethanol was able to act as a solvent in the reaction [18]. Methanol does not have a similar ability to act as solvent and therefore has lower reaction yields and higher waste production. This suggests that the diffusive process can be used to reduce the energy requirements for biodiesel production but excess solvents will be required.

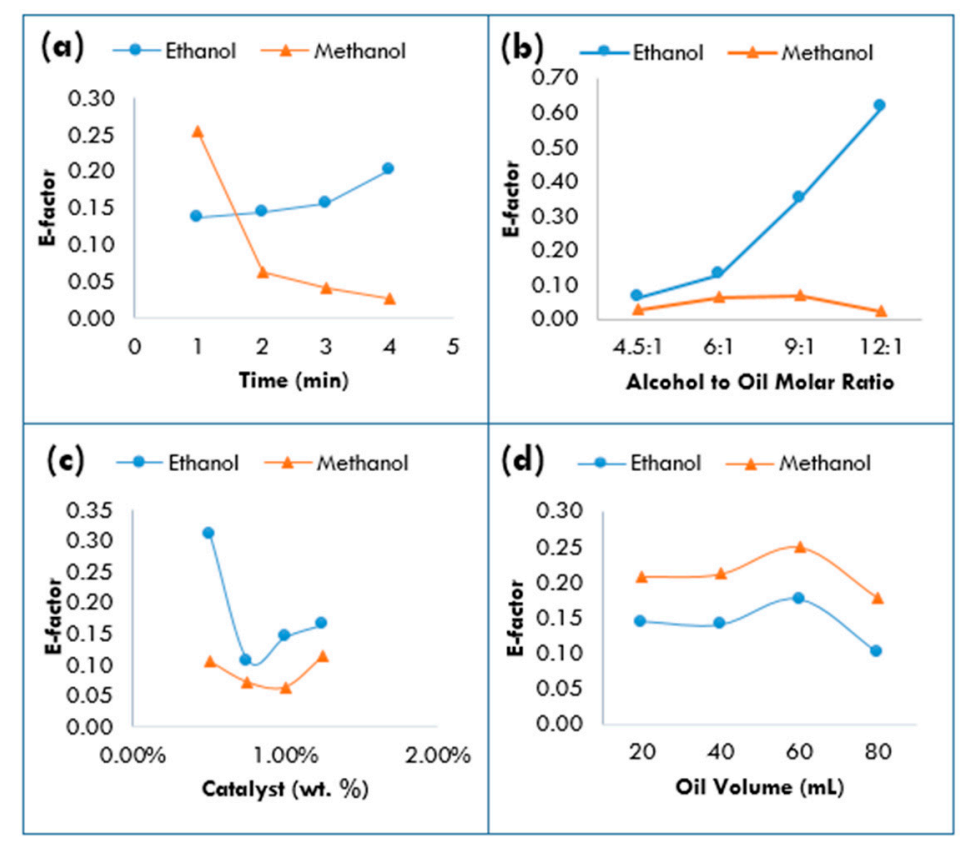

Figure 2. Environmental factor results for the simultaneous effect of microwave and ultrasound (MW/US) in the waste cooking oil (WCO) transesterification. Effect of (a) different reaction time; (b) alcohol to oil molar ratio; (c) catalyst concentration and (d) sample (oil) volume.

\subsection{Atom Economy (AE) or Atom Efficiency}

The molecular weights of biodiesel product, oil, catalyst, and alcohol were used to calculate the $A E$ for ethanol and methanol, which were $86 \%$ and $88 \%$ respectively. Even though atom economy is one of the most commonly used green metrics [21], a conclusion cannot be made from this result since it is only based on molecular weight.

Figure 3 shows the atom efficiency results based on biodiesel yields, and it was observed that methanol is more atom efficient than ethanol. This could be due to the fact that ethanol has a higher molecular weight and higher boiling point, and it requires higher temperature (energy) than methanol to react. The efficiency increases as the reaction time increases due to higher reaction temperatures and reaction exposure. However, it is not favorable when using low catalyst concentrations due to lower yield and lower atom economy. 


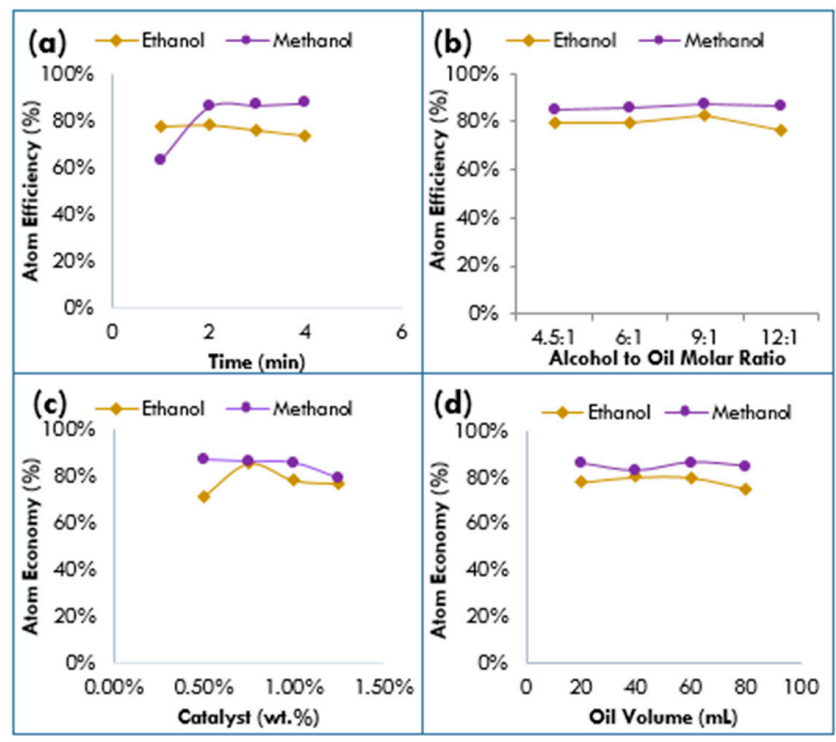

Figure 3. Atom efficiency results for the simultaneous effect of MW/US in WCO transesterification. Effect of (a) different reaction time; (b) alcohol to oil molar ratio; (c) catalyst concentration and (d) sample (oil) volume.

\subsection{Mass Intensity (MI) and Mass Productivity (MP)}

The results for $M I$ and $M P$ are shown in Figures 4 and 5, respectively. The process mass intensity is much higher when using ethanol, which means that the total mass used in the process is not recovered, and the mass of the final product is low. Despite this, the results revealed that the ideal $M I(1 \mathrm{~kg} / \mathrm{kg})$ was almost achieved by methanol; the MI, when using ethanol, was also close to unity. Therefore, both of these alcohols are green solutions or solvents to produce biodiesel, with methanol being better than ethanol. It should be noted that with higher alcohol ratios the mass productivity decreases for ethanol which mainly depends on the experimental yields achieved in this study.

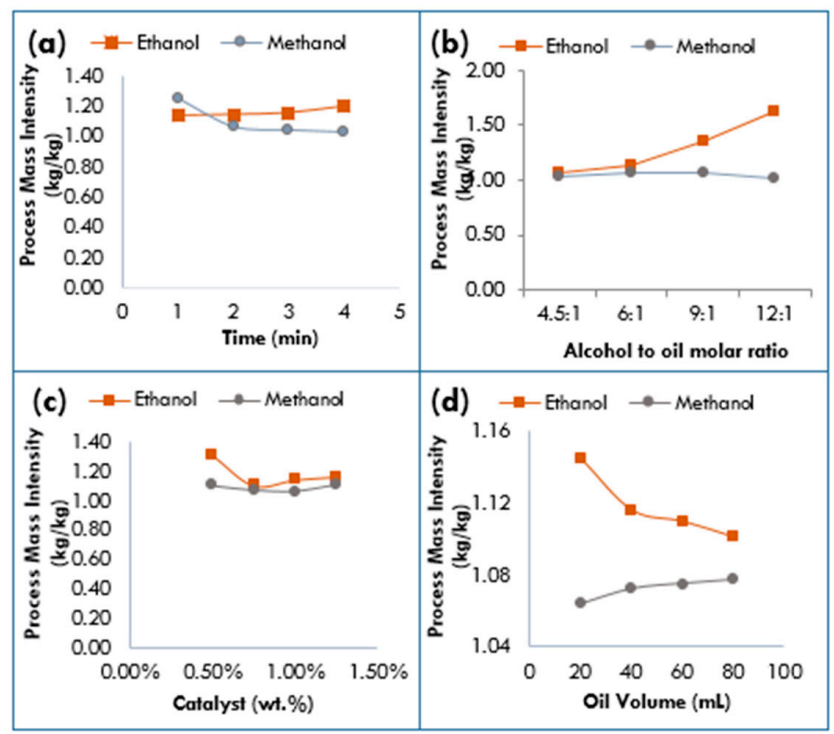

Figure 4. Mass Intensity results for the simultaneous effect of MW/US in WCO transesterification. Effect of (a) different reaction time; (b) alcohol to oil molar ratio; (c) catalyst concentration and (d) sample (oil) volume. 


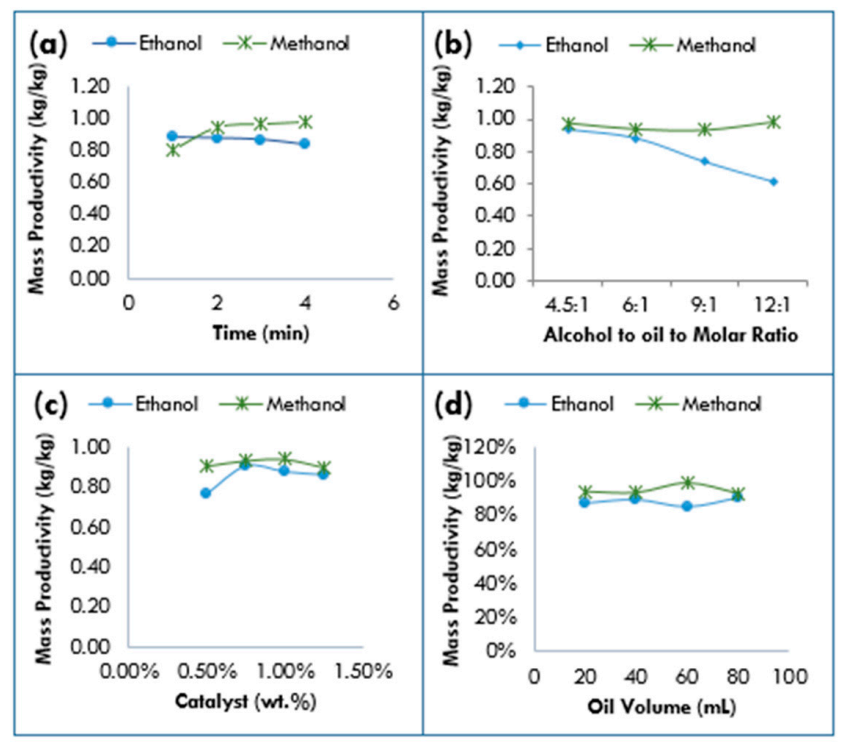

Figure 5. Mass Productivity for the simultaneous effect of MW/US in WCO transesterification. Effect of (a) different reaction time; (b) alcohol to oil molar ratio; (c) catalyst concentration and (d) sample (oil) volume.

\subsection{Reaction Mass Efficiency (RME) or Material Efficiency (ME)}

The results shown in Figures 6 and 7 were calculated using Equations (7) and (8), respectively. The results shown in Figure 7 are based on the E-factor, but a similar trend was followed using both equations. As shown in Figure 7, the reaction mass efficiency percentages were higher when based on the E-factor, which could be due to the fact the E-factor is solely based on the amount of waste produced. Therefore, more than one alternative is available to calculate RME. The results favored the transesterification reaction using methanol for both expressions. In addition, the differences between $M I$ and $M P$ values are due to the differences in environmental factors calculated in these reactions.

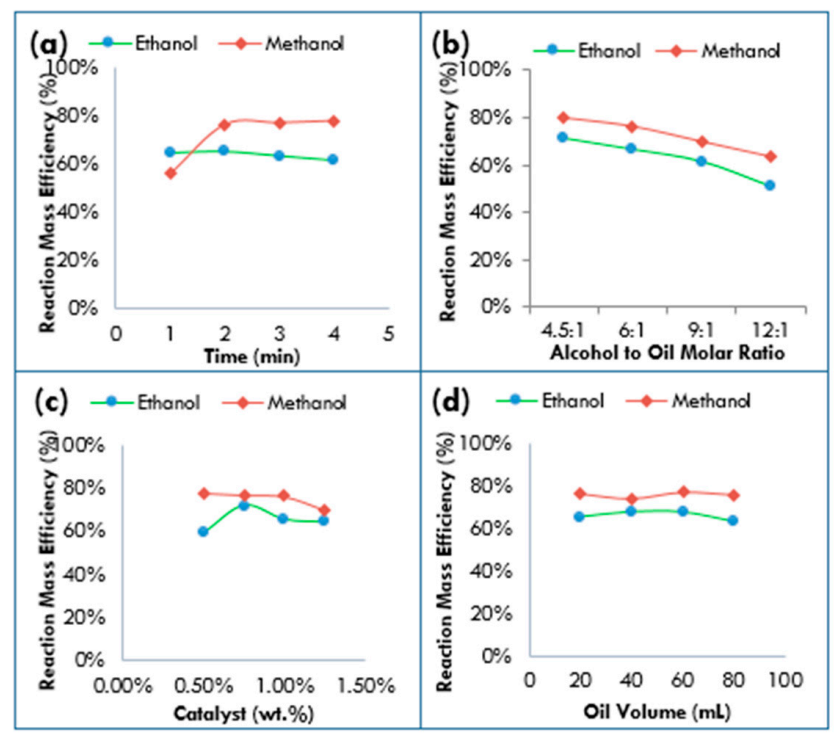

Figure 6. Reaction mass efficiency for the simultaneous effect of MW/US in WCO transesterification. Effect of (a) different reaction time; (b) alcohol to oil molar ratio; (c) catalyst concentration and (d) sample (oil) volume. 


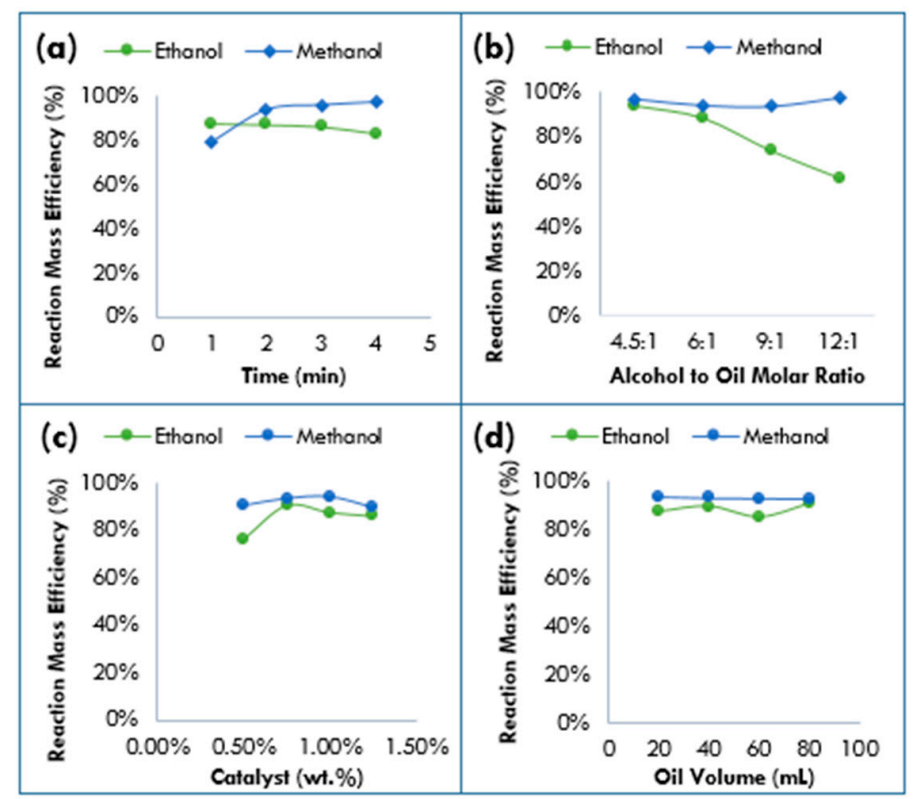

Figure 7. Reaction mass efficiency or Material efficiency for the simultaneous effect of MW/US in WCO transesterification. Effect of (a) different reaction time; (b) alcohol to oil molar ratio; (c) catalyst concentration and (d) sample (oil) volume.

\subsection{Atom Utilization}

Atom utilization was calculated using Equation (9) (see Figure 8). Since the atom utilization is based on the waste generated in reactions, the obtained results from the WCO conversion appear to be low; however, as previously stated, this is due to the fact that glycerol was considered a waste. Therefore, these results can be improved if a proper use is given to glycerol or it is considered as a beneficial product.

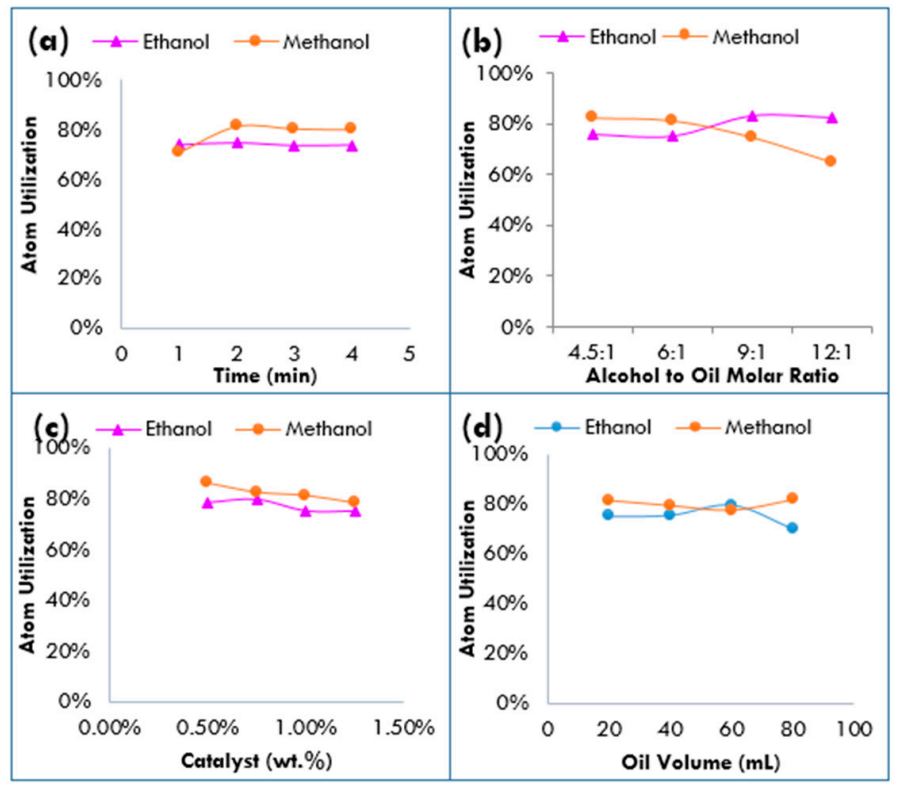

Figure 8. Atom utilization for the simultaneous effect of MW/US in WCO transesterification. Effect of (a) different reaction time; (b) alcohol to oil molar ratio; (c) catalyst concentration and (d) sample (oil) volume. 


\subsection{Solvent and Catalyst Environmental Impact Parameter $(f)$}

As shown in Figure 9, the solvent and catalyst environmental impact parameter was higher for ethanol due to its higher molecular weight of $(46.07 \mathrm{~g} / \mathrm{mol})$ when compared to methanol $(32.04 \mathrm{~g} / \mathrm{mol})$. In addition, mass of the final product using ethanol was lower because there was more waste produced in the process.

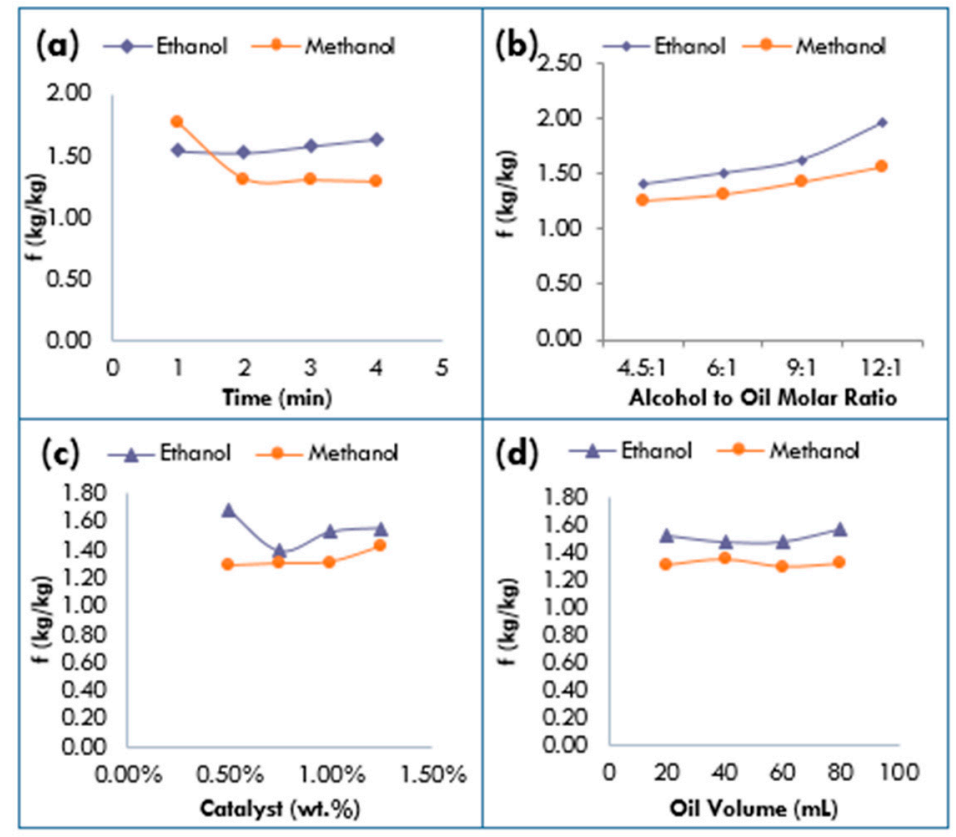

Figure 9. Solvent and catalyst environmental impact parameter (f) for the simultaneous effect of MW/US in WCO transesterification. Effect of (a) different reaction time; (b) alcohol to oil molar ratio; (c) catalyst concentration and (d) sample (oil) volume.

\subsection{Stoichiometric Factor}

As mentioned earlier, completion of the transesterification reaction requires excess quantities of solvents over the stoichiometric ratios. The ethanol and methanol to oil ratios used in the experimental studies were significantly higher (1.5 to 4 times) than the stoichiometric ratios which contributed to a higher stoichiometric factor for both solvents (see Table 1). Of these, it should be noted that ethanol has slightly higher values due to its higher molecular weight.

Table 1. Stoichiometric factor.

\begin{tabular}{ccc}
\hline Process Condition & Stoichiometric Factor Ethanol & Methanol \\
\hline $4.5: 1$ & 1.07 & 1.05 \\
$6: 1$ & 1.14 & 1.12 \\
$9: 1$ & 1.28 & 1.22 \\
$12: 1$ & 1.43 & 1.32 \\
\hline
\end{tabular}

\section{Conclusions and Future Directions}

The application of green chemistry metrics or sustainability indicators to biodiesel production has been investigated and quantified based on the experimental results derived in this study. Even though these metrics consist of simple calculations, they can provide a clear insight into the greenness of biodiesel production. From the results, it was noted that the reaction is more favorable or "green" or sustainable when using methanol as a reactant. However, ethanol has been referred to as a "green solvent" in many applications including biodiesel production due to its environmentally friendly 
characteristics. From the green metrics evaluations conducted in this study, it can be concluded that even though ethanol can be produced from renewable materials, its use does not necessarily make biodiesel production greener. Methanol as a reactant is not entirely desirable due to its nonrenewable nature and environmental impacts associated with its production. Therefore, it is suggested to further investigate the use of renewable feedstock to produce a methanol or microbial biomethanol production route. The production of biomethanol would not only be a contribution to sustainable biodiesel production, but also a viable alternative for other biofuel, biochemical, industrial and pharmaceutical processes that utilize methanol as a reactant or solvent.

Acknowledgments: The authors would like to acknowledge the partial funding support by The United States Environmental Protection Agency (USEPA) under the Grant number SU835519. No funds were received for covering the costs to publish in open access.

Author Contributions: E.M.G. and V.G.G. conceived and designed the experiments; E.M.G. performed the experiments; E.M.G. and V.G.G. analyzed the data; E.M.G. and V.G.G. wrote the paper.

Conflicts of Interest: The authors declare no conflict of interest. The funding sponsors had no role in the design of the study; in the collection, analyses, or interpretation of data; in the writing of the manuscript, and in the decision to publish the results.

\section{Appendix A}

Sample calculations for each metric:

Assuming: $6: 1$ ethanol to oil ratio, $1 \% \mathrm{NaOH}, 80 \mathrm{~mL}$ of oil, $20 \mathrm{~mL}$ of glycerol $(1.26 \mathrm{~g} / \mathrm{mL}$ density), $71 \mathrm{~mL}$ of biodiesel $(0.9 \mathrm{~g} / \mathrm{mL}$ density)

Find: each green metric for the sample

Solution:

Weight $=$ volume $\times$ density

Oil weight $=80 \mathrm{~mL} \times 0.92 \mathrm{~g} / \mathrm{mL}=73.6 \mathrm{~g}$ of oil

Ethanol weight $=33.39 \mathrm{~mL} \times 0.79 \mathrm{~g} / \mathrm{mL}=26.4 \mathrm{~g}$ of ethanol

Catalyst weight $=73.6 \mathrm{~g}$ of oil $\times 1 \% \mathrm{NaOH}=0.736 \mathrm{~g}$ of $\mathrm{NaOH}(w t / w t)$

Glycerol weight $=20 \mathrm{~mL} \times 1.26 \mathrm{~g} / \mathrm{mL}=25.2 \mathrm{~g}$ of glycerol

Biodiesel weight $=71 \mathrm{~mL} \times 0.9 \mathrm{~g} / \mathrm{mL}=63.9 \mathrm{~g}$ of biodiesel

\section{E-Factor:}

Mass in the process $=$ Oil + ethanol + catalyst $=73.6 \mathrm{~g}+26.4 \mathrm{~g}+0.736 \mathrm{~g}=100.736 \mathrm{~g}$

Products $=$ Glycerol + biodiesel $=25.2 \mathrm{~g}+63.9 \mathrm{~g}=89.1 \mathrm{~g}$

Waste $=100.736 \mathrm{~g}-89.1 \mathrm{~g}=11.636 \mathrm{~g}$

$E-$ factor $=\frac{\text { Total waste }(\mathrm{kg})}{\text { Product }(\mathrm{kg})}=0.011636 \mathrm{~kg} / 0.0891 \mathrm{~kg}=0.13$

E-factor $=0.13$

Atom Economy:

$$
A E=\frac{M W \text { of product } C}{M W \text { of } A+M W \text { of } B} \times 100
$$

Molecular weight: biodiesel $(856 \mathrm{~g} / \mathrm{mol})$, ethanol $(46.07 \mathrm{~g} / \mathrm{mol})$, catalyst $(39.997 \mathrm{~g} / \mathrm{mol})$, oil $(922 \mathrm{~g} / \mathrm{mol})$

$$
A E=\frac{856 \mathrm{~g} / \mathrm{mol}}{\left(922 \frac{\mathrm{g}}{\mathrm{mol}}+39.997 \frac{\mathrm{g}}{\mathrm{mol}}+46.07 \frac{\mathrm{g}}{\mathrm{mol}}\right)}=84.9 \%
$$

Atom Economy $=85 \%$

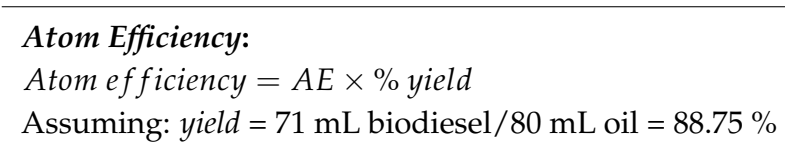

$$
\text { Atom efficiency }=84.9 \% \times 88.75 \%=75.3 \%
$$

Atom efficiency $=75 \%$ 
Mass Intensity $(M I)$ and Mass Productivity $(M P)$ :

$$
M I=\frac{\text { Total mass used in a process }(\mathrm{kg})}{\text { Mass of final product }(\mathrm{kg})}
$$

Mass used in the process $(\mathrm{kg})=0.100736$ (from E-factor calculation)

Mass of final product $(\mathrm{kg})=0.0891$

$$
\begin{gathered}
M I=\frac{0.100736 \mathrm{~kg}}{0.0891 \mathrm{~kg}}=1.13 \mathrm{~kg} / \mathrm{kg} \\
M P=\frac{1}{M I} \times 100 \\
M P=\frac{1}{1.13} \times 100=88.5 \%
\end{gathered}
$$

$M I=1.13 \mathrm{~kg} / \mathrm{kg}$ and $M P=88.5 \%$

$$
\begin{aligned}
& \text { Reaction Mass Efficiency: } \\
& R M E=\frac{\text { Mass of product }(C+D)(\mathrm{kg})}{\text { Mass of } A(\mathrm{~kg})+\text { mass of } B(\mathrm{~kg})} \\
& R M E=\frac{1}{1+E_{f}} \\
& R M E=\frac{0.0891 \mathrm{~kg}}{0.100736 \mathrm{~kg}}=88 \% \\
& R M E=\frac{1}{1+E_{f}}=\frac{1}{1+0.13}=88 \% \\
& A U \%=\frac{\text { Mass of the final product }}{\text { Total mass of all substances produced }} \\
& A U \%=\frac{63.9 g}{89.1 g}=72 \%
\end{aligned}
$$

$A U=72 \%$

\section{Solvent and Catalyst Environmental Impact Parameter:}

$$
\begin{gathered}
f=\frac{\sum \text { mass of reactants and postreaction solvents and materials }+ \text { mass of catalyst used }}{\text { Mass of final product }} \\
\qquad f=\frac{73.6+26.4+0.736+25.2}{63.9}=1.97
\end{gathered}
$$

$f=1.97 \mathrm{~kg} / \mathrm{kg}$

Stoichiometric Factor:

$$
S F=1+\frac{(A E) \sum \text { Mass of excess } \frac{\text { reagents }}{\text { chemicals }}}{\text { Expected product mass at } 100 \% \text { yield }}
$$

The ideal stoichiometry is a 3:1 alcohol to oil molar ratio. In these sample calculations, the used molar ratio is 6:1. Therefore, the ideal ethanol amount should be $16.7 \mathrm{~mL}(13.2 \mathrm{~g})$, but $33.39 \mathrm{~mL}(26.4 \mathrm{~g})$ was used instead. Expected product mass at $100 \%$ yield $=13.2+73.6+0.736=87.536 \mathrm{~g}$

$$
S F=1+\frac{(0.849)(13.2)}{87.536}=1.12
$$

$S F=1.12 \mathrm{~kg} / \mathrm{kg}$

\section{References}

1. Gude, V.G.; Martinez-Guerra, E. Green chemistry of microwave-enhanced biodiesel production. In Production of Biofuels and Chemicals with Microwave; Springer: Dordrecht, The Netherlands, 2015; pp. 225-250. 
2. Sheldon, R.A. Green and sustainable manufacture of chemicals from biomass: State of the art. Green Chem. 2014, 16, 950-963. [CrossRef]

3. Andraos, J.; Sayed, M. On the use of "green" metrics in the undergraduate organic chemistry lecture and lab to assess the mass efficiency of organic reactions. J. Chem. Educ. 2007, 84, 1004. [CrossRef]

4. Anastas, P.T.; Warner, J.C. Green Chemistry: Theory and Practice; Oxford University Press: Oxford, UK, 2000.

5. Wardencki, W.; Curyło, J.; Namiesśnik, J. Green Chemistry-Current and Future Issues. Pol. J. Environ. Stud. 2005, 14, 389-395.

6. Tobiszewski, M.; Marć, M.; Gałuszka, A.; Namieśnik, J. Green chemistry metrics with special reference to green analytical chemistry. Molecules 2015, 20, 10928-10946. [CrossRef] [PubMed]

7. Sheldon, R.A. Atom efficiency and catalysis in organic synthesis. Pure Appl. Chem. 2000, 72, 1233-1246. [CrossRef]

8. Constable, D.J.; Curzons, A.D.; Cunningham, V.L. Metrics to 'green' chemistry-Which are the best? Green Chem. 2000, 4, 521-527. [CrossRef]

9. Sheldon, R.A. Utilisation of biomass for sustainable fuels and chemicals: Molecules, methods and metrics. Catal. Today 2011, 167, 3-13. [CrossRef]

10. Manley, J.B.; Anastas, P.T.; Cue, B.W. Frontiers in Green Chemistry: Meeting the grand challenges for sustainability in R \& D and manufacturing. J. Clean. Prod. 2008, 16, 743-750.

11. Sheldon, R.A. The E factor: Fifteen years on. Green Chem. 2007, 9, 1273-1283. [CrossRef]

12. Dunn, P.J. The importance of green chemistry in process research and development. Chem. Soc. Rev. 2012, 41, 1452-1461. [CrossRef] [PubMed]

13. Dunn, P.J.; Galvin, S.; Hettenbach, K. The development of an environmentally benign synthesis of sildenafil citrate (Viagra ${ }^{\mathrm{TM}}$ ) and its assessment by Green Chemistry metrics. Green Chem. 2004, 6, 43-48. [CrossRef]

14. Jiménez-González, C.; Overcash, M.R. The evolution of life cycle assessment in pharmaceutical and chemical applications-A perspective. Green Chem. 2014, 16, 3392-3400. [CrossRef]

15. Gude, V.G. Synergism of microwaves and ultrasound for advanced biorefineries. Resour.-Effic. Technol. 2015, 1, 116-125. [CrossRef]

16. Martinez-Guerra, E.; Gude, V.G. Synergistic effect of simultaneous microwave and ultrasound irradiations on transesterification of waste vegetable oil. Fuel 2014, 137, 100-108. [CrossRef]

17. Martinez-Guerra, E.; Gude, V.G. Transesterification of waste vegetable oil under pulse sonication using ethanol, methanol and ethanol-methanol mixtures. Waste Manag. 2014, 34, 2611-2620. [CrossRef] [PubMed]

18. Martinez-Guerra, E.; Gude, V.G. Transesterification of used vegetable oil catalyzed by barium oxide under simultaneous microwave and ultrasound irradiations. Energy Convers. Manag. 2014, 88, 633-640. [CrossRef]

19. Trost, B.M. The atom economy-A search for synthetic efficiency. Science 1991, 254, 1471-1477. [CrossRef] [PubMed]

20. Welton, T. November. Solvents and sustainable chemistry. Proc. R. Soc. A 2015, 471, 20150502. [CrossRef] [PubMed]

21. Dicks, A.P.; Hent, A. Atom economy and reaction mass efficiency. In Green Chemistry Metrics; Springer: Berlin, Germany, 2015; pp. 17-44.

22. Andraos, J. Unification of reaction metrics for green chemistry: Applications to reaction analysis. Organ. Process Res. Dev. 2005, 9, 149-163. [CrossRef]

23. Andraos, J.; Dicks, A.P. Green chemistry teaching in higher education: A review of effective practices. Chem. Educ. Res. Pract. 2012, 13, 69-79. [CrossRef]

24. Gude, V.G.; Patil, P.; Martinez-Guerra, E.; Deng, S.; Nirmalakhandan, N. Microwave energy potential for biodiesel production. Sustain. Chem. Process. 2013, 1, 5. [CrossRef]

25. Helwani, Z.; Othman, M.R.; Aziz, N.; Fernando, W.J.N.; Kim, J. Technologies for production of biodiesel focusing on green catalytic techniques: A review. Fuel Process. Technol. 2009, 90, 1502-1514. [CrossRef]

26. Cesarini, S.; Pastor, F.I.; Nielsen, P.M.; Diaz, P. Moving towards a competitive fully enzymatic biodiesel process. Sustainability 2015, 7, 7884-7903. [CrossRef]

27. Gu, Y.; Jérôme, F. Glycerol as a sustainable solvent for green chemistry. Green Chem. 2010, 12, 1127-1138. [CrossRef] 
28. Kulkarni, M.G.; Gopinath, R.; Meher, L.C.; Dalai, A.K. Solid acid catalyzed biodiesel production by simultaneous esterification and transesterification. Green Chem. 2006, 8, 1056-1062. [CrossRef]

29. Wei, Z.; Xu, C.; Li, B. Application of waste eggshell as low-cost solid catalyst for biodiesel production. Bioresour. Technol. 2009, 100, 2883-2885. [CrossRef] [PubMed] 